\title{
3D-measurement of tracheobronchial angles on inspiratory and expiratory chest CT in COPD: respiratory changes and correlation with airflow limitation
}

This article was published in the following Dove Press journal:

International Journal of COPD

\author{
Rintaro Onoe' \\ Tsuneo Yamashiro 2,3 \\ Hiroshi Handa' \\ Shinya Azagami' \\ Shin Matsuoka \\ Takeo Inoue' \\ Teruomi Miyazawa' \\ Masamichi Mineshita' \\ 'Division of Respiratory Medicine, \\ Department of Internal Medicine, \\ St Marianna University School \\ of Medicine, Kawasaki, Japan; \\ ${ }^{2}$ Department of Radiology, \\ St Marianna University School \\ of Medicine, Kawasaki, Japan; \\ ${ }^{3}$ Department of Radiology, Graduate \\ School of Medical Science, University \\ of the Ryukyus, Nishihara, Japan
}

Purpose: To assess tracheobronchial angles and their changes on combined inspiratory and expiratory thoracic computed tomography (CT) scans and to determine correlations between tracheobronchial angles and several indices of chronic obstructive pulmonary disease (COPD).

Materials and methods: A total of 80 smokers underwent combined inspiratory and expiratory CT scans. Of these, 65 subjects also performed spirometry and 55 patients were diagnosed with COPD. On CT scans, 3-dimensinal tracheobronchial angles (trachea-right main bronchus [RMB], trachea-left main bronchus [LMB], and RMB-LMB) were automatically measured by software. Lung volumes at inspiration and expiration were also automatically calculated. Changes in tracheobronchial angles between inspiration and expiration were assessed by the Mann-Whitney test. Correlations of the angles with lung volume, airflow limitation, and CT-based emphysema index were evaluated by Spearman rank correlation.

Results: The trachea-LMB angle was significantly smaller and the RMB-LMB angle was significantly larger at expiration than inspiration $(P<0.0001)$. The trachea-LMB and RMBLMB angles were significantly correlated with lung volume, particularly at expiration. The RMB-LMB angle was significantly correlated with airflow limitation and CT emphysema index $(P<0.001-0.05)$ at inspiration and expiration, suggesting that narrowed RMB-LMB angle indicates more severe airflow limitation and larger extent of emphysema.

Conclusion: Tracheobronchial angles change during respiration and are correlated with severity of COPD and emphysema.

Keywords: tracheobronchial angle, computed tomography, chronic obstructive pulmonary disease, emphysema

\section{Introduction}

Since the 1950s, many studies have focused on the anatomy of the tracheobronchial tree. ${ }^{1-7}$ In general, the trachea is located at the height of the 6th cervical vertebra and branches to the left and right main bronchus (LMB and RMB) at the height of the 4-5th thoracic vertebra. A recent study analyzing chest computed tomography (CT) of Asian populations reported that the angle of the RMB is $35^{\circ}$ and that of the $\mathrm{LMB}$ is $43^{\circ} .{ }^{8}$ However, tracheobronchial angles vary by age, race, and individual, and the methodology of angle measurement can affect the results. ${ }^{8-16}$ Hence, it is difficult to confidently determine the "normal" tracheobronchial angles.

In previous studies, tracheobronchial angles were measured on chest radiographs or chest CT at full inspiration only. ${ }^{8,9,11,12,16}$ Thus, it is unclear how the tracheobronchial
Correspondence: Tsuneo Yamashiro Department of Radiology, Graduate School of Medical Science, University of the Ryukyus, 207 Uehara, Nishihara, Okinawa 903-0215, Japan

$\mathrm{Tel}+8 \mid 98895$ II 62

Fax $+8|98895| 420$

Email clatsune@yahoo.co.jp 
angles change from inspiration to expiration. Also, although CT scans were reconstructed into the coronal view, both chest radiographs and CT scans were analyzed as 2-dimensional (2D) images. Since the trachea descends obliquely on the lateral view of chest radiographs and the main bronchi branch in 3 dimensions, 3-dimensional (3D) measurements will provide "true" angles formed by the trachea and main bronchi.

It has been reported that cardiac expansion, such as cardiomegaly due to heart failure, enlarges the angle between the RMB and LMB. ${ }^{10,12}$ A recent series of CT studies analyzing heart size on combined inspiratory and expiratory CT scans reported that heart size increases from inspiration to expiration primarily due to elevation of the diaphragm during expiration. Furthermore, patients with chronic obstructive pulmonary disease (COPD) often had no change in heart size between inspiratory and expiratory CT scans because of limited lung volume (LV) change due to airflow limitation. ${ }^{17,18}$ From these observations, it can be predicted that the tracheobronchial angles must change during respiration and may correlate with COPD severity.

COPD and tracheobronchomalacia can be cited as diseases predicted to influence the tracheal bifurcation angle. In this study, we analyzed tracheobronchial angles and their changes during respiration using 3D techniques. We began with existing inspiratory and expiratory CT scans in smokers with and without COPD. The aims of our study were as follows: 1) to clarify whether or not the 3D-tracheobronchial angles change during respiration; 2) to investigate relationships between tracheobronchial angles and physiological factors such as LV; and 3) to determine if tracheobronchial angles correlate with indicators of COPD, such as airflow limitation and emphysema index.

\section{Materials and methods}

This retrospective study was approved by the Institutional Review Board of St Marianna University School of Medicine. Patients' consent to review their medical records was not required by the Institutional Review Board of St Marianna University School of Medicine since all medical records including $\mathrm{CT}$ scans were obtained for routine clinical care for the patients. All patients' data were anonymized for analyses.

\section{Subjects}

We reviewed the medical records and existing chest CT scans of all patients diagnosed with, or suspected to have, COPD, who underwent inspiratory and expiratory chest CT for assessment of emphysema or airway disease between January 2017 and April 2017. A total of 87 patients were identified, all of whom were ex- or current smokers. Seven patients were excluded from the study due to previous lung operation $(n=2)$, severe cardiomegaly $(n=3)$, and obvious pneumonia/atelectasis $(n=2)$. Ultimately, 80 patients (70 males and 10 females, with a mean age of 73 years [range, 41-87 years]) were included in the study. Among them, 65 subjects had performed spirometry and 55 were diagnosed with COPD (Figure 1). Patient characteristics are summarized in Table 1.

\section{CT scanning}

All patients were scanned using 64-row detector CT scanners (Aquilion 64; Canon Medical Systems, Otawara, Tochigi, Japan). Chest CT was performed in the supine position during breath hold at full inspiration and expiration. The scanning parameters were as follows: collimation, $0.5 \mathrm{~mm}$;

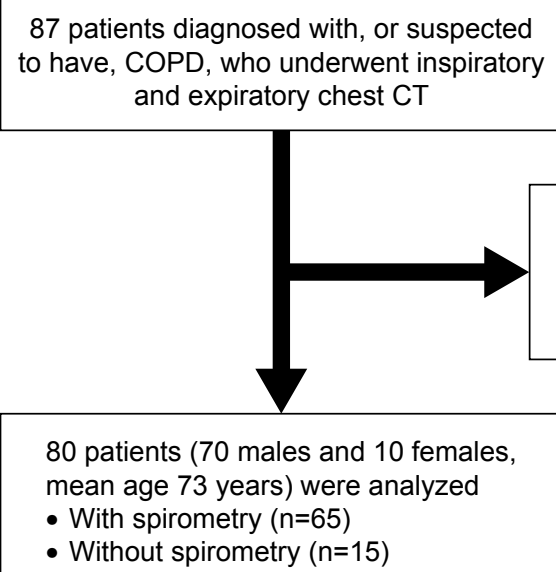

80 patients (70 males and 10 females,

palyzed

- Without spirometry $(n=15)$

7 patients were excluded:

- Previous lung operation $(n=2)$

- Severe cardiomegaly $(n=3)$

- And pneumonia/atelectasis $(n=2)$

Figure I A flowchart for patient selection.

Abbreviations: COPD, chronic obstructive pulmonary disease; CT, computed tomography. 
Table I Clinical characteristics and spirometric values

\begin{tabular}{|c|c|c|}
\hline Patient characteristics $(n=80)$ & Mean \pm SD & Range \\
\hline Sex (male:female) & $(70: 10)$ & \\
\hline Age (years) & $73 \pm 8$ & $(4 \mid-87)$ \\
\hline Height (cm) & $163 \pm 7$ & $(144-177)$ \\
\hline Weight (kg) & $60 \pm 10$ & $(3|-9|)$ \\
\hline $\operatorname{BSA}\left(m^{2}\right)$ & $1.65 \pm 0.15$ & $(1.15-2.05)$ \\
\hline Brinkman index $(n=74)$ & $\mathrm{I}, 257 \pm 642$ & $(200-2,800)$ \\
\hline \multicolumn{3}{|l|}{ Spirometric values $(n=65)$} \\
\hline FVC (L) & $3.13 \pm 0.87$ & $(1.37-6.32)$ \\
\hline $\mathrm{FEV}_{1}(\mathrm{~L})$ & $1.75 \pm 0.67$ & $(0.54-4.33)$ \\
\hline $\mathrm{FEV}_{\mathrm{l}} / \mathrm{FVC}(\%)$ & $56.5 \pm 16.0$ & $(22.5-98.5)$ \\
\hline $\mathrm{FEV}_{1}(\%$ predicted $)$ & $66.5 \pm 20.0$ & $(24 . I-I \mid 2.0)$ \\
\hline
\end{tabular}

Abbreviations: BSA, body surface area; $\mathrm{FEV}_{1}$, forced expiratory volume in I second; FVC, forced vital capacity; SD, standard deviation.

tube voltage, $120 \mathrm{kVp}$; tube current $200 \mathrm{~mA}$ (at inspiration) and $80 \mathrm{~mA}$ (at expiration); gantry rotation time, 0.5 seconds; beam pitch, 0.828 (53/64). All images were reconstructed using a standard kernel (for mediastinum) with a slice thickness of $0.5 \mathrm{~mm}$. The imaging field of view was $320 \mathrm{~mm}$, and the pixel size was $0.625 \times 0.625 \mathrm{~mm}$.

\section{$C T$ analysis - 3D measurements of the tracheobronchial angles}

Using research software based on commercially available software (4D Airways Analysis; Canon), 3D measurements of the tracheobronchial angles on chest CT were fully automatically obtained (Figure 2). In brief, the software

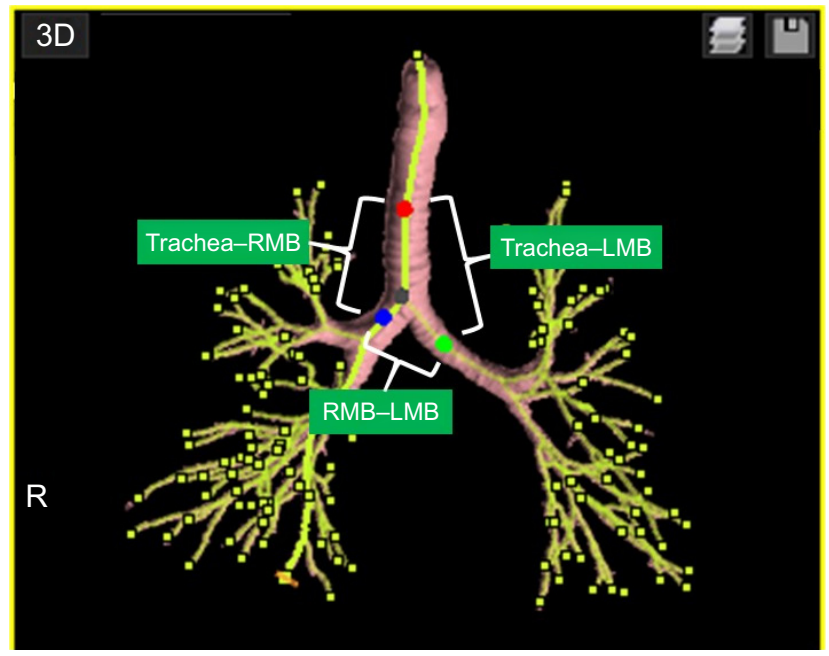

Figure 2 An example of tracheobronchial angle measurements by the research software.

Notes: On the yellow-colored airway center lines, 4 points were automatically identified: trachea (red), bifurcation (black), RMB (blue) and LMB (green). The tracheal point was set $40 \mathrm{~mm}$ above the bifurcation point. In this case, 3Dmeasurements of the 3 angles formed by the 4 points were as follows: trachea-RMB, I42.9 ; trachea-LMB, $137.8^{\circ}$; RMB-LMB, 79.4ㅇ.

Abbreviations: $L M B$, left main bronchus; RMB, right main bronchus. first identified the center lines of the tracheobronchial tree. On the center lines, a "tracheal point" was set $40 \mathrm{~mm}$ above the bifurcation (branching) point of the RMB and LMB. On the center lines of the RMB and LMB, points dividing the total lengths of the RMB and LMB were identified as RMB and LMB points. On the straight lines formed by the 4 points (trachea, bifurcation, RMB, and LMB), 3D measurements of the 3 angles (trachea-RMB, trachea-LMB, and RMB-LMB) were automatically measured. The straight lines formed by the 4 points were different from the original (curved) centerlines created by the software. The software was operated by a pulmonologist ( $\mathrm{RO}$, with 3 years of experience in respiratory medicine).

\section{CT analysis - emphysema and LV measurements}

As an index for the extent of pulmonary emphysema, the low attenuation volume percentage (LAV\%) was automatically measured using commercially available software (Lung Volume Measurement, Canon). ${ }^{19}$ In brief, the software segmented the lung parenchyma (between -500 and -1,024 HU) from the chest wall and the hilum, created a density histogram of the lung parenchyma, and calculated LV and LAV\% $(<-950 \mathrm{HU}) .{ }^{20-23} \mathrm{LV}$ was measured on both inspiratory and expiratory $\mathrm{CT}$ scans and LAV\% was measured on inspiratory CT scans only. In each subject, an operator (RO) confirmed that the software properly excluded the trachea and the proximal bronchi from the measured lung field. LV measurements were adjusted by body surface area (BSA) in each subject. ${ }^{24}$

\section{Spirometry}

Among the 80 subjects, 65 performed spirometry within 2 months of the CT exams. Spirometry was performed according to the protocol of the European Respiratory Society and American Thoracic Society. ${ }^{25}$ Measurements included FVC, forced vital capacity (FVC) and forced expiratory volume in 1 second $\left(\mathrm{FEV}_{1}\right)$. The ratio of $\mathrm{FEV}_{1}$ to $\mathrm{FVC}$ was calculated $\left(\mathrm{FEV}_{1} / \mathrm{FVC}\right)$. Values for $\mathrm{FEV}_{1}$ were also expressed as percent of predicted values.

\section{Statistical analysis}

Correlations between tracheobronchial angles, LV, and $\mathrm{LAV} \%$ measurements and spirometric values were determined using Spearman rank correlation analysis. Differences in inspiratory and expiratory values were assessed by the Mann-Whitney test. All statistical analyses were performed using JMP software, version 12.0 (SAS Institute, Cary, NC, 
USA). Continuous data were expressed as mean \pm standard deviation (SD). For all statistical analyses, a $P$-value $<0.05$ was considered statistically significant.

\section{Results}

\section{Patient characteristics}

Patient characteristics and spirometric values are shown in Table 1. The subjects ranged in age from 41 to 87 years (mean 73 years), and the Brinkman index (the number of cigarettes smoked per day multiplied by the number of years of smoking), which is often used as an index for the cumulative dose of smoking, ranged from 200 to 2,800 (mean 1,257, after excluding 6 subjects whose detailed smoking index was unclear). The mean spirometric values were as follows: $\mathrm{FEV}_{1}, 1.75 \pm 0.67 \mathrm{~L}(0.54-4.33$ $\mathrm{L}) ; \mathrm{FEV}_{1} / \mathrm{FVC}, 56.5 \% \pm 16.0 \%(22.5 \%-98.5 \%) ; \mathrm{FEV}_{1}$ (\%predicted), 66.5\% $20.0 \%(24.1 \%-112.0 \%)$. According to the Global Initiative for Chronic Obstructive Lung Disease staging, ${ }^{26} 55$ subjects were diagnosed with COPD (Global Initiative for Chronic Obstructive Lung Disease stage $1, n=7$; stage $2, n=24$; stage $3, n=22$; and stage 4 , $\mathrm{n}=2$ ). The remaining 10 subjects were smokers without COPD.

\section{Tracheobronchial angles}

Tracheobronchial angle measurements are shown in Table 2. The average trachea-RMB angle was $142.9^{\circ} \pm 10.1^{\circ}$ $\left(106.6^{\circ}-170.0^{\circ}\right)$ at inspiration and $144.7^{\circ} \pm 9.3^{\circ}\left(110.7^{\circ}-167.0^{\circ}\right)$ at expiration $(P<0.01)$. The average trachea-LMB angle was $141.5^{\circ} \pm 6.6^{\circ}\left(129.9^{\circ}-163.1^{\circ}\right)$ at inspiration and $136.6^{\circ} \pm 7.4^{\circ}$ $\left(117.0^{\circ}-157.5^{\circ}\right)$ at expiration $(P<0.0001)$. The average RMB-LMB angle was $74.4^{\circ} \pm 9.7^{\circ}\left(41.2^{\circ}-98.1^{\circ}\right)$ at inspiration and $77.0^{\circ} \pm 9.5^{\circ}\left(45.8^{\circ}-100.8^{\circ}\right)$ at expiration $(P<0.0001)$. The trachea-LMB angle was significantly smaller and the RMB-LMB angle was significantly larger at expiration than at inspiration (Figure 3).

\section{CT-based LV and emphysema index}

LV measurements are shown in Table 2. The average LV of the entire lung was $5.1 \pm 1.1 \mathrm{~L}(2.7-7.9 \mathrm{~L})$ at inspiration and $3.1 \pm 0.7 \mathrm{~L}(1.6-6.2 \mathrm{~L})$ at expiration $(P<0.0001)$. The LAV\% on inspiratory $\mathrm{CT}$ scans significantly correlated with $\mathrm{FEV}_{1} / \mathrm{FVC}(\rho=-0.64, P<0.0001)$. The LV of the entire lung, adjusted by BSA, also correlated with $\mathrm{FEV}_{1} / \mathrm{FVC}$ (at inspiration, $\rho=-0.44, P<0.001$; at expiration, $\rho=-0.55$, $P<0.0001)$.

\section{Correlation between tracheobronchial angles and CT-based LV}

The tracheobronchial angles and their correlations with LV are shown in Table 3. At inspiration, the trachea-LMB angle weakly correlated with whole and right $\mathrm{LV}$, adjusted by BSA $(P<0.05)$. The RMB-LMB angle weakly correlated with LV at inspiration $(P<0.05)$.

At expiration, the trachea-LMB angle was significantly and positively correlated with LV measurement adjusted by BSA $(\rho=0.57-0.61, P<0.0001)$. This indicates that larger LV at expiration, which implies hyperinflation and airflow limitation, causes the larger trachea-LMB angles.

Also at expiration, the RMB-LMB angle significantly and negatively correlated with LV measurement adjusted by BSA ( $\rho=-0.29$ to $-0.33, P<0.01)$. This suggests that larger $\mathrm{LV}$ at expiration leads to a smaller (sharper) RMB-LMB angle.

\section{Correlation of tracheobronchial angles with spirometric values and CT emphysema index}

Correlations between tracheobronchial angles and spirometric values and CT-based emphysema index are shown in Table 4. The RMB-LMB angle was significantly and positively correlated with $\mathrm{FEV}_{1} / \mathrm{FVC}$ and $\mathrm{FEV}_{1}$ (\% $\%$ predicted) at inspiration $(\rho=0.42$ and $0.39, P<0.001)$ and at expiration ( $\rho=0.38, P<0.01$ for both spirometric values) (Figure 4 ).

Table 2 Tracheobronchial angles and LV measurements

\begin{tabular}{|c|c|c|c|}
\hline CT measurement & Inspiration & Expiration & $P$-value ${ }^{a}$ \\
\hline \multicolumn{4}{|l|}{ Angle } \\
\hline Trachea-RMB $\left({ }^{\circ}\right)$ & $142.9 \pm 10.1(106.6-170.0)$ & $144.7 \pm 9.3(\mid 10.7-167.0)$ & $<0.01$ \\
\hline Trachea-LMB $\left({ }^{\circ}\right)$ & $14 \mid .5 \pm 6.6(|29.9-| 63.1)$ & $136.6 \pm 7.4(\mid 17.0-157.5)$ & $<0.000$ I \\
\hline RMB-LMB $\left(^{\circ}\right)$ & $74.4 \pm 9.7(41.2-98.1)$ & $77.0 \pm 9.5(45.8-100.8)$ & $<0.0001$ \\
\hline \multicolumn{4}{|l|}{ LV } \\
\hline Whole lung (L) & $5.1 \pm 1.1(2.7-7.9)$ & $3.1 \pm 0.7(1.6-6.2)$ & $<0.0001$ \\
\hline Right lung (L) & $2.7 \pm 0.6(1.6-4.2)$ & $1.7 \pm 0.4(1.0-3.4)$ & $<0.000$ I \\
\hline Left lung (L) & $2.3 \pm 0.6(1.1-3.7)$ & $1.4 \pm 0.4(0.6-3.1)$ & $<0.0001$ \\
\hline
\end{tabular}

Note: ${ }^{a}$ Comparisons between inspiratory and expiratory values were made using the Wilcoxon matched-pairs test.

Abbreviations: CT, computed tomography; LMB, left main bronchus; LV, lung volume; RMB, right main bronchus. 

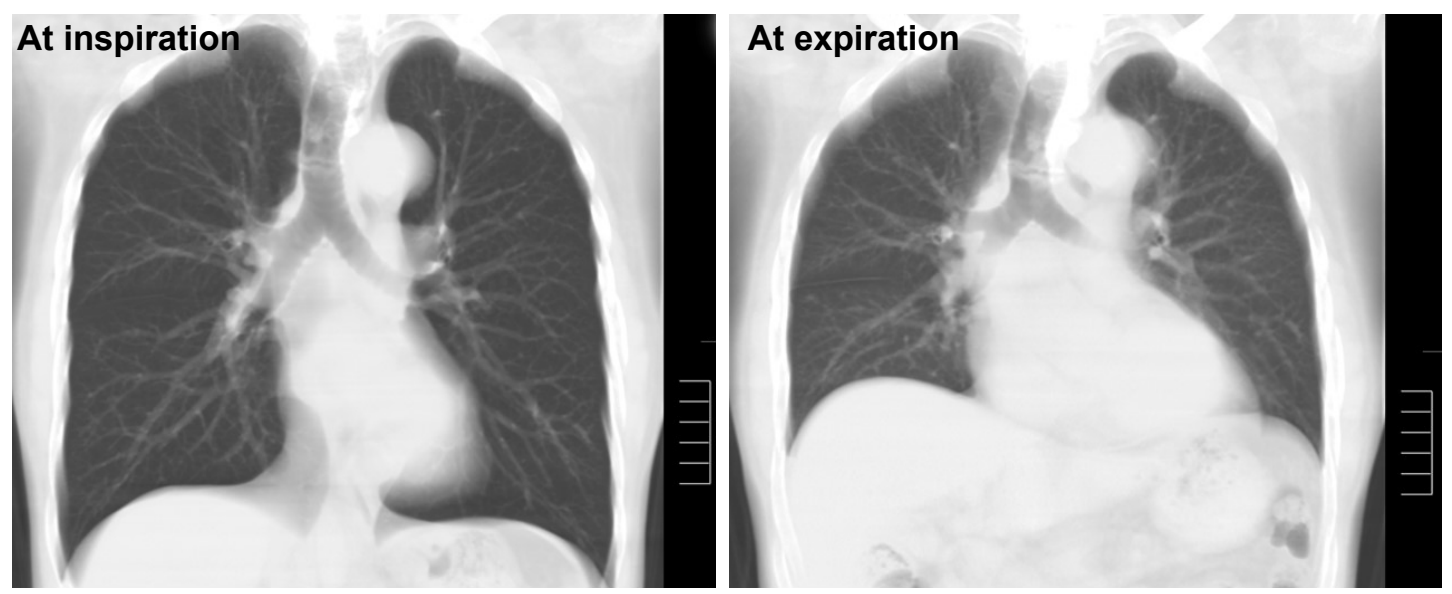

Figure 3 Reconstructed coronal CT images of a 72-year-old male smoker with mild COPD (FEV $/$ FVC $=66 \%$, LAV\%= $13 \%$ ).

Notes: At expiration (right), the diaphragm moves upward and the tracheobronchial angles change from those at inspiration (left). Angle measurements were as follows: trachea-RMB, $144.8^{\circ}$ (inspiration), $140.7^{\circ}$ (expiration); trachea-LMB, $143.9^{\circ}$ (inspiration), $129.2^{\circ}$ (expiration); RMB-LMB, $71.2^{\circ}$ (inspiration), $83.0^{\circ}$ (expiration).

Abbreviations: COPD, chronic obstructive pulmonary disease; CT, computed tomography; $\mathrm{FEV}_{1}$, forced expiratory volume in I second; FVC, forced vital capacity; LAV, low attenuation volume; LMB, left main bronchus; RMB, right main bronchus.

The trachea-LMB angle was significantly and negatively correlated with $\mathrm{FEV}_{1} / \mathrm{FVC}$ and $\mathrm{FEV}_{1}$ (\%predicted) at expiration $(\rho=-0.38, P<0.01$ for both) (Figure 5).

At both inspiration and expiration, the RMB-LMB angle was significantly and negatively correlated with LAV\% ( $\rho=-0.27$ to $-0.29, P<0.05)$. The expiratory trachea-LMB angle was significantly and positively correlated with LAV\% $(\rho=0.34, P<0.01)$.

These data suggest that larger (wider) RMB-LMB angles are associated with less severe airflow limitation and emphysema both at inspiration and at expiration, and that smaller (sharper) trachea-LMB angles at expiration are associated with less severe airflow limitation and emphysema. However, the trachea-RMB angle rarely correlates with airflow limitation or emphysema severity.

Table 3 Correlations between tracheobronchial angles and LV measurements

\begin{tabular}{|c|c|c|c|}
\hline \multirow{2}{*}{$\begin{array}{l}\text { Angle } \\
\text { measurement }\end{array}$} & \multicolumn{3}{|l|}{ LV/BSA } \\
\hline & Whole lung & Right lung & Left lung \\
\hline \multicolumn{4}{|l|}{ Inspiration } \\
\hline \multicolumn{4}{|l|}{ Angle } \\
\hline Trachea-RMB & 0.09 (NS) & 0.05 (NS) & 0.11 (NS) \\
\hline Trachea-LMB & $0.25(P<0.05)$ & $0.28(P<0.05)$ & 0.21 (NS) \\
\hline RMB-LMB & $-0.26(P<0.05)$ & $-0.22(P<0.05)$ & $-0.26(P<0.05)$ \\
\hline \multicolumn{4}{|l|}{ Expiration } \\
\hline \multicolumn{4}{|l|}{ Angle } \\
\hline Trachea-RMB & -0.06 (NS) & -0.07 (NS) & -0.04 (NS) \\
\hline Trachea-LMB & $0.60(P<0.000 \mathrm{I})$ & $0.57(P<0.000 \mathrm{I})$ & $0.61(P<0.0001)$ \\
\hline RMB-LMB & $-0.33(P<0.01)$ & $-0.29(P<0.0 \mathrm{I})$ & $-0.37(P<0.001)$ \\
\hline
\end{tabular}

Abbreviations: BSA, body surface area; LMB, left main bronchus; LV, lung volume; NS, not significant; RMB, right main bronchus.

\section{Discussion}

In this study, we used 3D CT images at inspiration and expiration and found that 1) tracheobronchial angles significantly change between inspiration and expiration; 2) the RMB-LMB and trachea-LMB angles are significantly correlated with $\mathrm{LV}$, whereas the trachea-RMB angle is not correlated with LV; and 3) the RMB-LMB and trachea-LMB angles are significantly correlated with spirometric values and CT-based emphysema index (smaller RMB-LMB angles and larger trachea-LMB angles are correlated with more severe COPD). Although the study population was limited to current or ex-smokers with COPD or suspected to have COPD, we believe that our observations provide an understanding of the

Table 4 Correlations between tracheobronchial angles and COPD indices

\begin{tabular}{|c|c|c|c|}
\hline \multirow{2}{*}{$\begin{array}{l}\text { Angle } \\
\text { measurement }\end{array}$} & \multicolumn{2}{|l|}{ Spirometry } & \multirow{2}{*}{$\begin{array}{l}\text { Emphysema } \\
\text { index } \\
\text { LAV\% (whole } \\
\text { lung) }(n=80)\end{array}$} \\
\hline & $\begin{array}{l}\text { FEV,IFVC } \\
(n=65)\end{array}$ & $\begin{array}{l}\text { FEV } \text { (\%predicted) } \\
(n=65)\end{array}$ & \\
\hline \multicolumn{4}{|l|}{ Inspiration } \\
\hline \multicolumn{4}{|l|}{ Angle } \\
\hline Trachea-RMB & -0.24 (NS) & $-0.25(P<0.05)$ & 0.10 (NS) \\
\hline Trachea-LMB & -0.14 (NS) & -0.12 (NS) & 0.18 (NS) \\
\hline RMB-LMB & $0.42(P<0.00 I)$ & $0.39(P<0.00 I)$ & $-0.29(P<0.05)$ \\
\hline \multicolumn{4}{|l|}{ Expiration } \\
\hline \multicolumn{4}{|l|}{ Angle } \\
\hline Trachea-RMB & -0.16 (NS) & -0.2 (NS) & $0.1 \mathrm{I}$ (NS) \\
\hline Trachea-LMB & $-0.38(P<0.0 \mathrm{I})$ & $-0.38(P<0.0 \mathrm{I})$ & $0.34(P<0.0 \mathrm{I})$ \\
\hline RMB-LMB & $0.38(P<0.01)$ & $0.38(P<0.01)$ & $-0.27(P<0.05)$ \\
\hline
\end{tabular}

Abbreviations: COPD, chronic obstructive pulmonary disease; $\mathrm{FEV}_{1}$, forced expiratory volume in I second; FVC, forced vital capacity; LAV\%, low attenuation volume percent $(<-950 \mathrm{HU})$; LMB, left main bronchus; NS, not significant; $\mathrm{RMB}$, right main bronchus. 

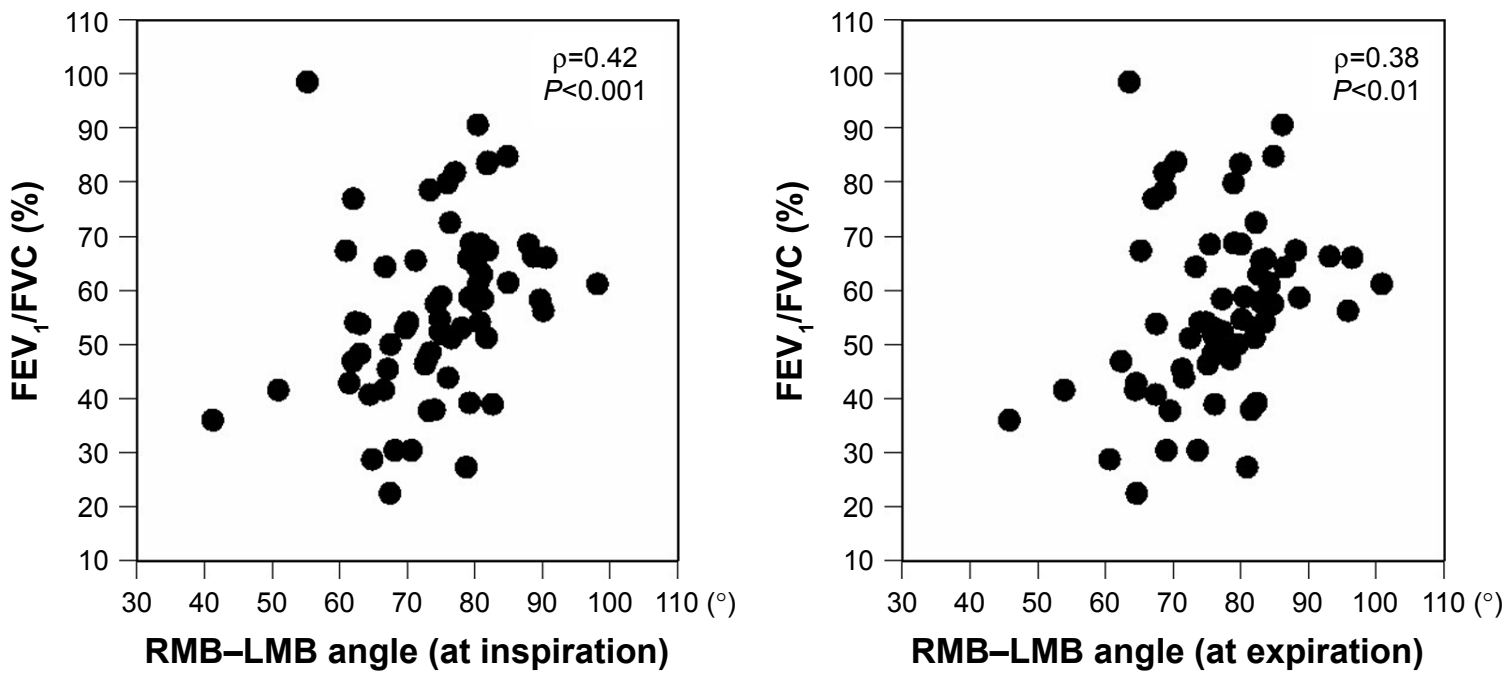

Figure 4 Correlations between tracheobronchial angles and spirometric values.

Notes: The angles formed by the right and left main bronchi (RMB-LMB) at inspiration and expiration significantly correlate with spirometric values (FEV/FVC).

Abbreviations: $F E V_{1}$, forced expiratory volume in I second; FVC, forced vital capacity; LMB, left main bronchus; RMB, right main bronchus.

physiological movements of the trachea and proximal bronchi in all patients.

It has been unclear whether, and to what extent, tracheobronchial angles change between inspiration and expiration, since all previous studies utilizing medical images analyzed only inspiratory chest radiographs or CT images.$^{3-5,8-13}$ Here, we analyzed both inspiratory and expiratory CT scans and found that tracheobronchial angles significantly changed during respiration. Although respiratory changes in the
trachea-RMB angle were relatively small, the trachea-LMB angle changed by $\sim 5^{\circ}$ (decreased by expiration) and the RMB-LMB angle changed by $2.6^{\circ}$ (increased by expiration). Considering the significant correlations between LV and these 2 angles, it is clear that the trachea-LMB and RMB-LMB angles are influenced by respiration. Although it is difficult to precisely identify physiologic reasons for the respiratory changes in tracheobronchial angles, the following explanation is plausible: from inspiratory to expiratory
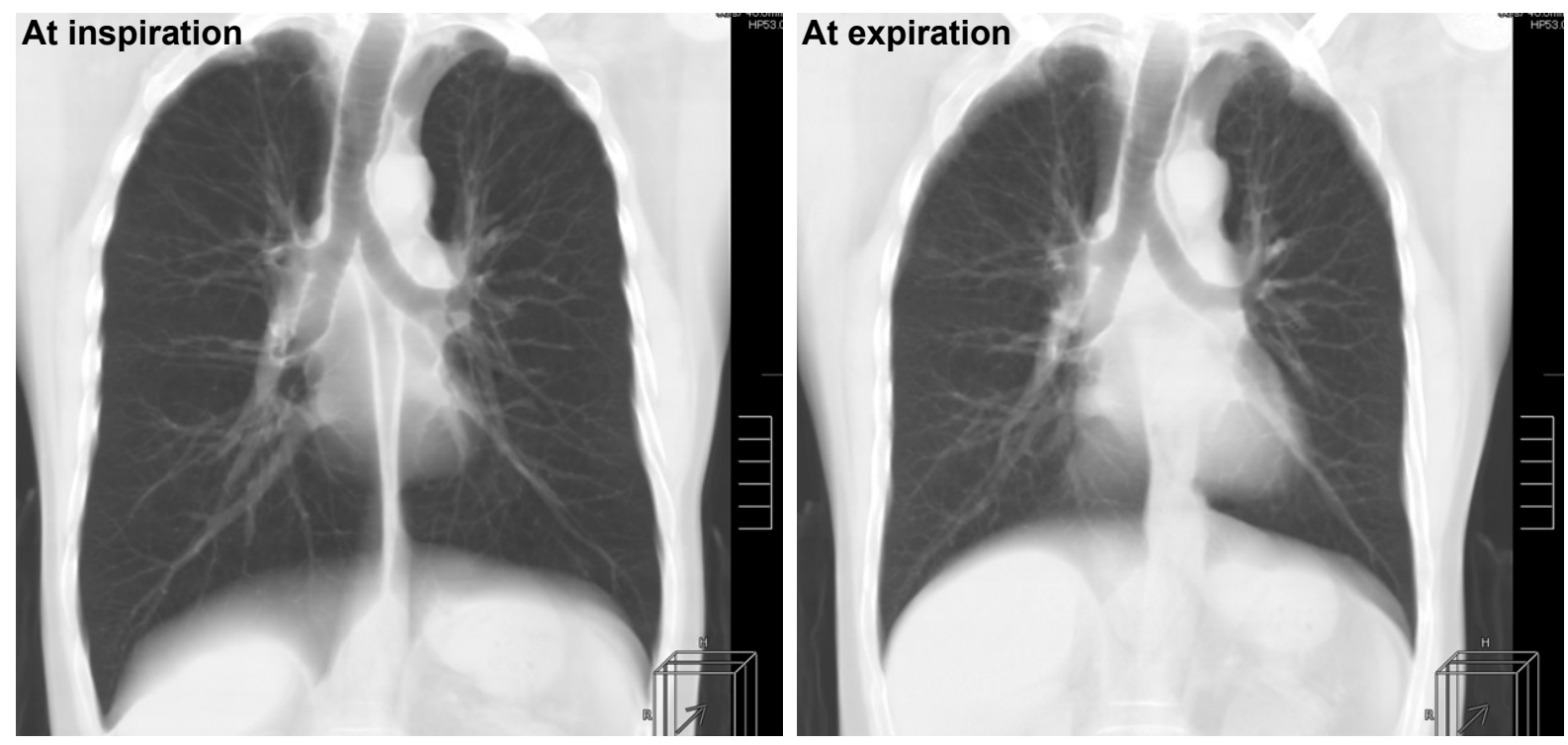

Figure 5 Reconstructed coronal CT images of a 54-year-old male with severe COPD (FEV,/FVC=42\%, LAV\%=43\%).

Notes: Compared with a mild COPD patient (shown in Figure 2), lung size and location of the diaphragm are not largely changed from inspiration (left) to expiration (right), suggesting the presence of severe air-trapping and airflow limitation. Tracheobronchial angles are similar on inspiratory and expiratory images. Angle measurements: trachea-RMB, $165.9^{\circ}$ (inspiration), $163.9^{\circ}$ (expiration); trachea-LMB, $141.0^{\circ}$ (inspiration), $141.5^{\circ}$ (expiration); RMB-LMB, 50.9 (inspiration), 53.9 ${ }^{\circ}$ (expiration).

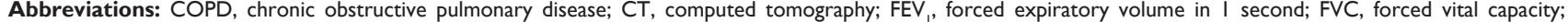
LAV, low attenuation volume; LMB, left main bronchus; RMB, right main bronchus. 
phases, the diaphragm elevates upward and the lower lobes decrease in size. Furthermore, the heart size increases in the horizontal direction, ${ }^{17,18}$ which compresses the tracheal bifurcation similar to cardiomegaly. ${ }^{10,12}$ These physiological changes result in widening of the tracheal bifurcation, which leads to an increased RMB-LMB angle and a decreased trachea-LMB angle. Compared with the LMB, the RMB is very short and is more distant to the heart. Thus, the tracheaRMB angle may be minimally changed by respiration, which would explain the insignificant correlation to LV in this study. This explanation is speculative and should be reevaluated by future studies with more subjects, with and without morbid conditions of the lung.

This study also clarified that, in addition to cardiomegaly or heart failure, ${ }^{10,12}$ COPD affects the tracheobronchial angles. Based on the observations showing significant correlations between the tracheobronchial angles and the LV in this study, it is relatively easy to predict correlations between tracheobronchial angles and airflow limitation or emphysema severity. As COPD worsens due to progressive emphysema and air-trapping, the lung becomes hyperinflated. The diaphragm is compressed downward and flattened. Furthermore, airflow limitation and air-trapping causes insufficient expiration and minimum elevation of the diaphragm during expiratory scanning. These changes will lead to larger LV at inspiration and expiration in patients with severe COPD, which underlies the significant correlations between the tracheobronchial angles (trachea-LMB and RMB-LMB) and spirometric values and CT emphysema index.

Although some studies have analyzed the trachea and proximal bronchi on expiratory $\mathrm{CT}$ and dynamic-ventilation $\mathrm{CT},{ }^{21-23}$ the clinical implications of understanding tracheal morphology have been limited. However, our observations of tracheobronchial angles, particularly regarding changes with respiration, can be used in the clinical setting. For example, preoperative planning of endotracheobronchial stenting is a good indication for 3D measurement of tracheobronchial angles. This can be applied in patients with excessive dynamic airway collapse related to COPD, relapsing polychondritis, and tracheobronchial stenosis due to malignant neoplasms. ${ }^{27-29}$ Although a previous study precisely analyzed branching angles of the RMB and LMB on chest CT to simulate insertion of double-lumen endotracheal tubes for lung isolation (1-lung ventilation), ${ }^{30}$ no attempt has been made to modify the angles of endotracheobronchial stents. Currently, all commercially available endotracheobronchial stents (Y-shaped stents) have fixed angles (trachea-RMB angle $=135^{\circ}$, trachea $-\mathrm{LMB}$ angle $=150^{\circ}$ ), which are not suitable for certain patients. Unfitted, fixed angle endotracheobronchial stents may cause secondary airway stenosis due to inflammatory granuloma formation. We found that the tracheobronchial angles vary within a population and that respiratory changes in the angles are not small. In the future, to prepare "best-fit" endotracheobronchial stents in advance of stenting, 3D measurements of the tracheobronchial angles on inspiratory and expiratory CT may play an important role and can inform 3D printing of stents and the tracheobronchial tree itself. If the patient has large respiratory changes in the tracheobronchial angles on $\mathrm{CT}$, stents made from a flexible matrix may be chosen. Although it is not common to scan patients at expiration (except for patients with obstructive disease), our study suggests that it may be useful to perform inspiratory and expiratory $\mathrm{CT}$ scans in patients scheduled to receive endotracheobronchial stenting. However, it should also be noted that excessive dynamic airway collapse related to COPD also occurs more peripheral airways than relapsing polychondritis or tracheobronchial stenosis caused by tuberculosis, which may not be treated by endotracheobronchial stenting.

This study has several limitations. First, all study subjects had COPD or were smokers with suspected COPD. Thus, respiratory changes in the tracheobronchial angles in healthy subjects and in patients with other lung diseases remain unknown. Also, spirometric values were not available for 15 subjects, which resulted in heterogeneous study population in this study. We believe that our approach would be acceptable for the first assessment of respiratory changes in the tracheobronchial angles; however, future researches should include COPD patients only or enroll normal controls to make a more precise conclusion regarding the influence of COPD on the tracheobronchial angles. Second, this was a male-dominant study cohort with a relatively small number of subjects $(n=80)$, which may have skewed the results. Third, the enrolled subjects were Japanese only. Other ethnic groups may have different tracheobronchial angles. Fourth, although previous studies analyzed 2D medical images, we used a $3 \mathrm{D}$ approach. Hence, the RMB-LMB angle in this study may not be directly comparable with the RMB-LMB angle measured in previous studies. Also, the trachea-RMB angle in this study cannot be compared with the branching angle of the RMB in previous studies, which was often measured from a line extending to the bronchus intermedius. Fifth, we did not use lobar segmentation to assess the LV of each lobe. Since the distribution of emphysema may have been different among COPD patients, lobar volumes and their difference may have influenced the tracheobronchial angle values. Sixth, since this 
study mainly focused on physiological changes of the tracheobronchial angles, the clinical impact of our observations in routine care of COPD is limited.

\section{Conclusion}

In conclusion, we measured 3D tracheobronchial angles on inspiratory and expiratory CT scans and found that all angles significantly change during respiration and the RMB-LMB and trachea-LMB angles correlated with LV, airflow limitation, and emphysema index.

\section{Acknowledgment}

The authors thank Tatsuya Kimoto, MS (Canon Medical Systems) for his support in preparing the software.

\section{Disclosure}

Tsuneo Yamashiro received a research grant from Canon Medical Systems. The authors report no other conflicts of interest in this work.

\section{References}

1. Jesseph JE, Merendino KA. The dimensional interrelationships of the major components of the human tracheobronchial tree. Surg Gynecol Obstet. 1957;105(2):210-214.

2. Wailoo MP, Emery JL. Normal growth and development of the trachea. Thorax. 1982;37(8):584-587.

3. Griscom NT. Computed tomographic determination of tracheal dimensions in children and adolescents. Radiology. 1982;145(2):361-364.

4. Gamsu G, Webb WR. Computed tomography of the trachea and mainstem bronchi. Semin Roentgenol. 1983;18(1):51-60.

5. Breatnach E, Abbott GC, Fraser RG. Dimensions of the normal human trachea. AJR Am J Roentgenol. 1984;142(5):903-906.

6. Thurlbeck A, Horsfield K. Branching angles in the bronchial tree related to order of branching. Respir Physiol. 1980;41(2):173-181.

7. Horsfield K, Cumming G. Angles of branching and diameters of branches in the human bronchial tree. Bull Math Biophys. 1967;29(2): 245-259.

8. Mi W, Zhang C, Wang H, et al. Measurement and analysis of the tracheobronchial tree in Chinese population using computed tomography. PLoS One. 2015;10(4):e0123177.

9. Haskin PH, Goodman LR. Normal tracheal bifurcation angle: a reassessment. Am J Roentgenol. 1982;139(5):879-882.

10. Coppola V, Vallone G, Coscioni E, et al. [Normal value of the tracheal bifurcation angle and correlation with left atrial volume]. Radiol Med. 1998;95(5):461-465. Italian.

11. Karabulut N. CT assessment of tracheal carinal angle and its determinants. Br J Radiol. 2005;78(933):787-790.

12. Murray JG, Brown AL, Anagnostou EA, Senior R. Widening of the tracheal bifurcation on chest radiographs: value as a sign of left atrial enlargement. AJR Am J Roentgenol. 1995;164(5):1089-1092.

13. Herek D, Herek O, Ufuk F. Tracheobronchial angle measurements in children: an anthropometric retrospective study with multislice computed tomography. Clin Exp Otorhinolaryngol. 2017;10(2):188-192.
14. Daroszewski M, Szpinda M, Flisiński P, et al. Tracheo-bronchial angles in the human fetus: an anatomical, digital, and statistical study. Med Sci Monit Basic Res. 2013;19:194-200.

15. Kubota Y, Toyoda Y, Nagata N, et al. Tracheo-bronchial angles in infants and children. Anesthesiology. 1986;64(3):374-376.

16. Cleveland RH. Symmetry of bronchial angles in children. Radiology. 1979;133(1):89-93.

17. Tomita H, Yamashiro T, Matsuoka S, Matsushita S, Nakajima Y. Correlation between heart size and emphysema in patients with chronic obstructive pulmonary disease: CT-based analysis using inspiratory and expiratory scans. Chron Respir Dis. Epub 2017 Jan 1.

18. Tomita H, Yamashiro T, Matsuoka S, Matsushita S, Kurihara Y, Nakajima Y. Changes in cross-sectional area and transverse diameter of the heart on inspiratory and expiratory chest CT: correlation with changes in lung size and influence on cardiothoracic ratio measurement. PLoS One. 2015;10(7):e0131902.

19. Sakai N, Mishima M, Nishimura K, Itoh H, Kuno K. An automated method to assess the distribution of low attenuation areas on chest CT scans in chronic pulmonary emphysema patients. Chest. 1994;106(5): 1319-1325.

20. Yamashiro T, Matsuoka S, Bartholmai BJ, et al. Collapsibility of lung volume by paired inspiratory and expiratory CT scans: correlations with lung function and mean lung density. Acad Radiol. 2010;17(4): 489-495.

21. Yamashiro T, San José Estépar R, Matsuoka S, et al. Intrathoracic tracheal volume and collapsibility on inspiratory and end-expiratory CT scans correlations with lung volume and pulmonary function in 85 smokers. Acad Radiol. 2011;18(3):299-305.

22. Usuba A, Yamashiro T, Handa H, et al. Quantitative computed tomography measurement of tracheal cross-sectional areas in relapsing polychondritis: correlations with spirometric values. Respiration. 2015; 90(6):468-473.

23. Yamashiro T, Moriya H, Tsubakimoto M, Matsuoka S, Murayama S. Continuous quantitative measurement of the proximal airway dimensions and lung density on four-dimensional dynamic-ventilation CT in smokers. Int J Chron Obstruct Pulmon Dis. 2016;11(1):755-764.

24. Du Bois D, Du Bois EF. A formula to estimate the approximate surface area if height and weight be known. Nutrition. 1989;5(5):303-311.

25. Laszlo G. Standardisation of lung function testing: helpful guidance from the ATS/ERS Task Force. Thorax. 2006;61(9):744-746.

26. Rabe KF, Hurd S, Anzueto A, et al. Global strategy for the diagnosis, management, and prevention of chronic obstructive pulmonary disease: GOLD executive summary. Am J Respir Crit Care Med. 2007;176(6): $532-555$.

27. Murgu S, Colt H. Tracheobronchomalacia and excessive dynamic airway collapse. Clin Chest Med. 2013;34(3):527-555.

28. Represas-Represas C, Leiro-Fernández V, Mallo-Alonso R, BotanaRial MI, Tilve-Gómez A, Fernández-Villar A. Excessive dynamic airway collapse in a small cohort of chronic obstructive pulmonary disease patients. Ann Thorac Med. 2015;10(2):118-122.

29. Azagami S, Yamashiro T, Handa H, et al. Tracheal stenosis caused by thoracic malignancy: correlation between CT-based tracheal measurement and spirometric values before and after tracheal stenting. Open J Med Imaging. 2017;7(1):63-76.

30. Patel RV, Van Noord BA, Patel D, et al. Determination of the true inclination angle of the main bronchi relative to the median sagittal plane for placement of a left-sided double-lumen tube. J Cardiothorac Vasc Anesth. 2017;31(2):434-440. 
International Journal of COPD

\section{Publish your work in this journal}

The International Journal of COPD is an international, peer-reviewed journal of therapeutics and pharmacology focusing on concise rapid reporting of clinical studies and reviews in COPD. Special focus is given to the pathophysiological processes underlying the disease, intervention programs, patient focused education, and self management protocols.

This journal is indexed on PubMed Central, MedLine and CAS. The manuscript management system is completely online and includes a very quick and fair peer-review system, which is all easy to use. Visit http://www.dovepress.com/testimonials.php to read real quotes from published authors 